\title{
Temporal turnover in the composition of tropical tree communities: functional determinism and phylogenetic stochasticity
}

\author{
Nathan G. Swenson, ${ }^{1,10}$ James C. Stegen, ${ }^{2,3}$ Stuart J. Davies, ${ }^{4,5}$ David L. Erickson, ${ }^{6}$ Jimena Forero-Montaña, ${ }^{7}$ \\ Allen H. Hurlbert, ${ }^{2}$ W. John Kress, ${ }^{6}$ Jill Thompson, ${ }^{7,8}$ María Uriarte, ${ }^{9}$ S. Joseph Wright, ${ }^{5}$ \\ AND JeSS K. ZimMERMAN ${ }^{7}$ \\ ${ }^{1}$ Department of Plant Biology, Michigan State University, East Lansing, Michigan 48824 USA \\ ${ }^{2}$ Department of Biology, University of North Carolina, Chapel Hill, North Carolina 27599 USA \\ ${ }^{3}$ Pacific Northwest National Laboratory, Fundamental and Computational Sciences Directorate, Biological Sciences Division, \\ Richland, Washington 99352 USA \\ ${ }^{4}$ Center for Tropical Forest Science, Arnold Arboretum, Harvard University, Cambridge, Massachusetts 02138 USA \\ ${ }^{5}$ Smithsonian Tropical Research Institute, P.O. Box 0843-03092, Balboa Ancón, Republic of Panama \\ ${ }^{6}$ Department of Botany, MRC-166, National Museum of Natural History, Smithsonian Institution, P.O. Box 37012, \\ Washington, D.C. 20013-7012 USA \\ ${ }^{7}$ Institute for Tropical Ecosystem Studies, University of Puerto Rico, Rio Piedras, Puerto Rico 00931 USA \\ ${ }^{8}$ Centre for Ecology and Hydrology, Edinburgh, Bush Estate, Penicuik, Midlothian, Scotland EH26 OQB United Kingdom \\ ${ }^{9}$ Department of Ecology, Evolution and Environmental Biology, Columbia University, New York, New York 10027 USA
}

Abstract. The degree to which turnover in biological communities is structured by deterministic or stochastic factors and the identities of influential deterministic factors are fundamental, yet unresolved, questions in ecology. Answers to these questions are particularly important for projecting the fate of forests with diverse disturbance histories worldwide. To uncover the processes governing turnover we use species-level molecular phylogenies and functional trait data sets for two long-term tropical forest plots with contrasting disturbance histories: one forest is older-growth, and one was recently disturbed. Having both phylogenetic and functional information further allows us to parse out the deterministic influences of different ecological filters. With the use of null models we find that compositional turnover was random with respect to phylogeny on average, but highly nonrandom with respect to measured functional traits. Furthermore, as predicted by a deterministic assembly process, the older-growth and disturbed forests were characterized by less than and greater than expected functional turnover, respectively. These results suggest that the abiotic environment, which changes due to succession in the disturbed forest, strongly governs the temporal dynamics of disturbed and undisturbed tropical forests. Predicting future changes in the composition of disturbed and undisturbed forests may therefore be tractable when using a functional-trait-based approach.

Key words: beta diversity; community dynamics; community phylogenetics; functional ecology; functional traits; neutral theory; Panama; Puerto Rico; tropical forest.

\section{INTRODUCTION}

A central challenge in community ecology is to determine the mechanisms that govern the structure and dynamics of biological communities (Hubbell 2001, Chase and Leibold 2003). Perhaps nowhere is this challenge greater than in tropical tree communities (Wright 2002), which contain large numbers of species with low population sizes. Mechanisms proposed to explain the dynamics of tropical tree communities vary along a continuum from deterministic (niche-based; Levine and HilleRisLambers 2009) to stochastic (dispersal-limitation and ecological drift; Hubbell 2001). Deterministic processes are expected to produce patterns

Manuscript received 29 June 2011; revised 19 August 2011; accepted 1 September 2011. Corresponding Editor: D. S. Srivastava.

${ }^{10}$ E-mail: swensonn@msu.edu of species turnover that are nonrandom with respect to species' functional ecology and, if function is evolutionarily conserved, phylogenetic relationships. If stochastic processes dominate, turnover is expected to be random with respect to both species' function and phylogenetic relationships.

The relative degree to which tropical tree community dynamics are deterministic or stochastic is impossible to quantify from temporal changes in species composition alone. This is because species names do not convey critical information regarding the ecological and evolutionary similarity of species (Webb 2000, Swenson et al. 2007, 2011, Swenson and Enquist 2009, Swenson 2011a). For example, complete turnover in the species composition of a community could result in a functionally analogous community, a directional change in the functional composition of a community, or changes in the community composition that are random with 
respect to species function (Swenson et al. 2011). For robust inferences to be made it is critical that analyses of community turnover go beyond the traditional approach of analyzing turnover in species composition by incorporating information pertaining to the ecological and evolutionary similarity of species.

Here, we directly quantify the degree to which the temporal turnover in tropical tree communities is deterministic or stochastic with respect to functional and phylogenetic similarity. However, examining raw levels of functional trait (or phylogenetic) turnover through time does not allow clear inferences to be made regarding the influences of stochastic and deterministic processes. One must instead examine rates of functional or phylogenetic turnover relative to the expected degree of functional or phylogenetic turnover. Here, the expected degree of functional or phylogenetic turnover is based on observed species turnover. Less than expected functional turnover, for example, is therefore a smaller change in functional trait composition than expected given the number of species that have changed. The expected degree of turnover is found by applying a null model that maintains the observed degree of species turnover, but randomizes the functional traits of and phylogenetic relatedness among species.

A stochastic model of community dynamics predicts that species turnover in the community will be random with respect to species function and phylogenetic position. That is, the magnitudes of observed functional and phylogenetic turnover will match their expected magnitudes, given the observed magnitude of species turnover. In contrast, a deterministic model of community dynamics related to the fit of organisms to the abiotic environment predicts that species turnover will be nonrandom with respect to species function in one of two ways: (1) if the local abiotic environment is relatively constant (e.g., in late-successional forests), community membership should be constrained such that there is less than expected temporal change in the functional composition of a community; or (2) if the local environment is changing relatively quickly (e.g., following an acute disturbance), the functional composition of a community should change faster than expected. Turnover in phylogenetic composition should show analogous patterns, but only if there is phylogenetic signal in species function.

Importantly, phylogenetic signal does not need to occur in measured traits for phylogenetic turnover to be nonrandom. As long as there is phylogenetic signal in ecologically important aspects of species function (measured or not), deterministic processes are expected to cause nonrandom phylogenetic turnover. In cases where there is little phylogenetic signal in measured traits, examining phylogenetic turnover can therefore provide additional information not provided by turnover patterns in measured functional traits. In particular, key defense traits are not often measured in traitbased community ecology, but have been found to have significant phylogenetic signal (Gilbert and Webb 2007). In situations where measured traits have little phylogenetic signal, we can therefore derive three additional predictions based on the action of deterministic processes. First, if unmeasured defense traits are the primary drivers of community dynamics, we expect random turnover in measured traits and nonrandom phylogenetic turnover. A prominent hypothesis for how defense traits influence community dynamics proposes that species with similar defense strategies will negatively influence each other by increasing the probability of attack by their shared pathogens (Janzen 1970, Connell 1971). In this case we specifically predict that an important influence of defense traits will lead to faster than expected phylogenetic turnover due to the shared enemies of closely related species governing the probability of recruitment and survival. If phylogenetic turnover is nonrandom, but is slower than expected in late-successional forests, this would indicate that other types of unmeasured traits (i.e., nondefense traits) influence community dynamics. Second, if measured traits are the primary drivers of community dynamics, we expect nonrandom functional turnover and random phylogenetic turnover. As discussed previously, we specifically predict that in this case functional turnover will be slower than expected in late successional forests and faster than expected in early-successional forests. We further predict that regions within a forest that were more recently disturbed than other parts of the same forest will show the fastest rates of functional trait turnover within that forest (relative to the null model). Likewise, the "oldest" regions within a forest should show the slowest rates of functional turnover (relative to the null model) within that forest. Third, if both measured traits and unmeasured defense traits have important influences over community dynamics, we expect nonrandom functional and phylogenetic turnover.

The present study tests these predictions in two longterm forest dynamics plots in order to gain a better understanding of the processes that govern the composition of tropical forests. The two specific forests studied here represent early- and late-successional forests. Both of these forests have areas within them that have variable degrees of disturbance allowing us to compare entire forests with differing levels of disturbance and to compare areas within each forest that have different levels of disturbance. Combining long-term data on the dynamics of their composition with species-level molecular phylogenies and functional traits provides a unique opportunity to test the nuances of predictions related to the dominance of deterministic or stochastic processes.

\section{Materials And Methods}

\section{Study sites}

The above predictions were tested in the 50-ha Barro Colorado Island Forest Dynamics Plot (BCI-FDP; Condit 1998, Hubbell et al. 1999, 2005) in Panama 
and the 16-ha Luquillo Forest Dynamics Plot (LUQFDP; Thompson et al. 2002) in Puerto Rico. Each forest plot has been censused multiple times where all freestanding woody stems $\geq 1 \mathrm{~cm}$ in diameter at breast height (dbh; $1.3 \mathrm{~m}$ above the ground) are identified, measured, and mapped. The BCI-FDP, which contains $\sim 300$ species at any given time, was originally censused in 1982 and every five years since 1985 with the most recent census in 2005. The vast majority of the BCI-FDP has been forested for at least the past 1000 years (Piperno 1989) with a small section being $\sim 110$ years old (Harms et al. 2001). The LUQ-FDP, which contains $\sim 130$ species at any given time, has been censused every five years since 1990 with the most recent census conducted in 2005 (Thompson et al. 2002). Parts of the LUQ-FDP were subject to logging and other human uses up until the 1940s and the entire LUQ-FDP was heavily disturbed by Hurricane Hugo in 1989 and Hurricane Georges in 1998 (Thompson et al. 2002). Thus the disturbance history of the LUQ-FDP provides a contrast to the less disturbed BCI-FDP that is critical for evaluating the generality of underlying processes. The two forest plots share an identical sampling protocol where during each census all individuals with a $\mathrm{dbh} \geq 1 \mathrm{~cm}$ are identified, mapped, and measured.

\section{Luquillo and BCI trait sampling methodology}

The functional trait measurements were generally performed in accordance with the universal protocols outlined by Cornelissen et al. (2003). There were three cases where our methods did not follow Cornelissen et al. (2003). First, when quantifying leaf traits, sun exposed leaves could not always be sampled because many species are only found in the shaded understory. In such cases leaf traits were collected from the crown of large individuals. Second, the seed mass measurements included endosperm and embryo only, while Cornelissen et al. (2003) only use diaspores. Third, wood density for shrubs could not be measured on main stems without killing individual plants. Thus, wood density was measured using branch material as described in Swenson and Enquist (2008). Over 90\% of the species had trait values determined by averaging the values taken from 15 or more individuals. The remaining rare species had trait values determined from the average of values taken from one to 15 individuals. The following traits were quantified: leaf area (LA), leaf percentage carbon $(\% \mathrm{C})$, leaf percentage nitrogen $(\% \mathrm{~N})$, leaf percentage phosphorus $(\% \mathrm{P})$, maximum height, seed mass, specific leaf area (SLA), and wood density (WD). These traits represent fundamental axes of ecological strategy in trees (Westoby et al. 2002, Swenson and Enquist 2007, Chave et al. 2009, Moles et al. 2009). The WD was measured using an increment borer in trees $10-20 \mathrm{~cm}$ in diameter at $1 \mathrm{~m}$ off the ground located outside of the LUQ-FDP. Maximum heights were taken by measuring the largest individual in the LUQ-FDP. Seeds were collected from seed traps placed within the LUQ-FDP from which their dry mass could be recorded. Although the leaf and wood trait data sets are complete for the LUQ-FDP, seed mass of 38 rare species could not be quantified due to their absence from seed traps.

The functional trait measurements for the BCI-FDP are described in Wright et al. (2010). Their collection protocols also follow Cornelissen et al. (2003) except for the first two exceptions described previously for the LUQ-FDP. Leaf traits were collected from the six largest individuals of each species. Rare species $(n<6)$ were not included in the BCI-FDP trait inventory.

\section{Functional trait dendrogram}

In order to apply identical analytical methods to the trait and phylogenetic data we constructed a functional trait dendrogram depicting functional similarity. This served as an analog to a phylogenetic tree depicting phylogenetic relatedness. The dendrogram for each plot was constructed by using the location of species' along the first three principal coordinate analysis (PCA) axes. The PCA analyses were performed because some of the measured traits were correlated. The PCA was computed using normally distributed trait data (normality achieved via transformation) that were transformed into standard normal deviates prior to PCA analysis. The PCA loadings and variance explained by each axis are provided in Appendix A. A Gower distance (Gower 1971) was used to generate a functional distance matrix that represented the functional similarity between species. A Gower distance was used because it allows for missing data in a species by trait data matrix. This distance matrix and group average clustering (UPGMA) were used to generate the functional dendrogram in the software " $\mathrm{R}$ " using the "stats" package (R Development Core Team 2010).

\section{$D N A$ sequencing and phylogenetic inference}

An exhaustive description of the methods used in both forests for DNA extraction, amplification, and sequencing are provided in Kress et al. $(2009,2010)$. The phylogenetic inference methods are briefly described here. The rbcL and matK sequences were globally aligned using Sequencer 4.8 (GeneCodes, Ann Arbor, Michigan, USA) and transalign (Binida-Edmonds 2005), respectively. The trnH-psbA sequences were aligned within families using Muscle (Edgar 2004) and concatenated to the rbcL+matK matrix to make a nested supermatrix.

The aligned sequence data for each gene were imported into MacClade and then concatenated. For the BCI-FDP we reconstructed the "community phylogeny" with parsimony using PAUP (Swofford 2003). For the LUQ-FDP a similar method was used except an ordinal-level constraint tree derived from the Angiosperm Phylogeny Group III phylogeny was utilized (Kress et al. 2010). 


\section{Quantifying phylogenetic signal}

We measured phylogenetic signal in the collected functional trait data and species abundances using the molecular phylogenies and Blomberg's $K$ statistic (Blomberg et al. 2003). Values of $K<1$ generally indicate a lack of phylogenetic signal and values $>1$ generally indicate significant phylogenetic signal. The R package "ape" was used to measure this statistic.

\section{Quantifying community phylogenetic and functional turnover}

The functional and phylogenetic compositional turnover of subplots in both forests was quantified using a pairwise dissimilarity metric (Swenson et al. 2011, Swenson 2011b):

$$
D_{\mathrm{PW}}=f_{A} \sum_{i=1}^{S_{A}} f_{i} \overline{\delta_{i b}}+f_{B} \sum_{j=1}^{S_{B}} f_{j} \overline{\delta_{j a}}
$$

where $S_{A}$ is the number of species in the community at time $A, S_{B}$ is the number of species in the community at time $B, f_{i}$ is the relative abundance of species $i$ in the community at time $A, f_{j}$ is the relative abundance of species $j$ in the community at time $B, \overline{\delta_{i b}}$ is the mean pairwise phylogenetic or functional distance between species $i$ in the community at time $A$ and all species in the community at time $B$, and $\overline{\delta_{j a}}$ is the mean pairwise phylogenetic or functional distance between species $j$ in the community at time $B$ and all species in the community at time $A$. So that $D_{\mathrm{PW}}$ was entirely based on phylogenetic or functional distances among individuals, relative community abundances were used to weight the contribution of each community to $D_{\mathrm{PW}}$. The relative abundance of the community at time $A, f_{A}$, was defined as the total number of individuals at time $A$ divided by the total number of individuals in the community at time $A$ and $B$. Pairwise phylogenetic and functional distances were calculated using the phylogenetic tree and dendrogram branch lengths, respectively. A null model was used that randomized species names across the tips of the phylogeny or dendrogram and calculated a null $D_{\mathrm{PW}}$ score 10000 times for each subplot. Thus, the null model randomized the phylogenetic relatedness and functional similarity of species, while maintaining the observed species abundance, species richness, species occupancy rates, and species turnover through time. It is also important to note that this null model procedure did not randomize the spatial distribution of individuals. Therefore it fixed the observed patterns of dispersal limitation of species. This null modeling procedure generated a distribution of 10000 null values for each subplot to which the observed $D_{\mathrm{PW}}$ value was compared.

Alternative null model approaches were considered, but none were finally implemented. Null models that randomize the community data matrix such as an independent swap algorithm were not chosen because they likely would result in stems changing species identity and traits between censuses, which is biologically unrealistic. Null models designed to constrain the randomization of species on the tips of the phylogeny into classes of similar abundance were considered. Such a null model is recommended when there is phylogenetic signal in species abundances. We tested for phylogenetic signal in species abundances and found none so there was no need to implement this null (Hardy 2008).

\section{Statistical analyses}

For the analyses, each forest dynamics plot was divided into nonoverlapping $400-\mathrm{m}^{2}$ subplots. Recent work from the LUQ-FDP and the BCI-FDP regarding spatial scale and spatial turnover in species composition and neighborhood interactions suggests that $400 \mathrm{~m}^{2}$ is an appropriate scale for the present study (Swenson et al. 2006, Zimmerman et al. 2010). The functional trait dendrogram and phylogenetic tree were used to quantify turnover in functional and phylogenetic diversity between the initial census and each subsequent census for each subplot using the phylogenetic and functional turnover and dispersion metrics described previously. The species turnover inside the $400-\mathrm{m}^{2}$ subplots was also calculated for comparison. This was quantified using a Bray-Curtis distance.

The number of species and individuals can bias raw measures of phylogenetic and functional diversity and their turnover making it difficult to determine the underlying ecological processes producing the observed pattern. Thus a null model was used that controlled for the observed turnover of species in each subplot. The null model analysis evaluates the null hypothesis that phylogenetic or functional turnover was random for each subplot given the observed turnover in species for that subplot (see Materials and methods: Quantifying community phylogenetic...).

The general goal of the present work was to detect whether or not the average phylogenetic and functional compositional turnover in the two forests has been nonrandom. This focus on the average turnover could potentially result in a lack of detection of habitat specific turnover trends. To investigate this possibility we quantified whether the rate of phylogenetic and functional turnover within the two plots significantly varied between habitat types. To do this we utilized previously published habitat-type designations for the BCI-FDP (Harms et al. 2001) and LUQ-FDP (Thompson et al. 2002). The BCI-FDP habitat types correspond more closely to topography, while the LUQ-FDP habitat types correspond to the human disturbance history of the forest (Appendix B). We used ANOVAs and Tukey tests to compare the distributions of habitat-specific phylogenetic and functional turnover values.

\section{Results \\ Phylogenetic signal}

For each of the plant functional traits in both forests $K$ values were much less than one, indicating little to no 
TABLE 1. Results from a test for phylogenetic signal in the functional trait data from the Barro Colorado Island (BCI) Forest Dynamics Plot (Panama) and Luquillo Forest Dynamics Plot (Puerto Rico), using the $K$ statistic proposed by Blomberg et al. (2003).

\begin{tabular}{lcc}
\hline \hline \multirow{2}{*}{\multicolumn{1}{c}{ Trait }} & \multicolumn{2}{c}{$K$ statistic } \\
\cline { 2 - 3 } & BCI & Luquillo \\
\hline $\log _{10}$ (maximum height) & 0.03 & 0.060 \\
$\log _{10}$ (leaf area) & 0.05 & 0.100 \\
Leaf \% & 0.03 & 0.010 \\
Leaf \% & 0.09 & 0.050 \\
Leaf \%P & 0.04 & 0.080 \\
$\log _{10}$ (seed mass) & 0.07 & 0.130 \\
$\log _{10}$ (specific leaf area) & 0.02 & 0.060 \\
Wood specific gravity & 0.03 & 0.070 \\
$\log _{10}$ (abundance in first census) & 0.099 & 0.144 \\
\hline
\end{tabular}

Note: Values of $K>1$ indicate phylogenetic signal in trait data.

phylogenetic signal in the data (Table 1). There was also little to no phylogenetic signal in species abundances in both plots (Table 1), which was important for null model selection.

\section{Species, phylogenetic, and functional temporal turnover}

The primary goal of this study was to examine the degree to which the compositional turnover of the two forests was nonrandom with respect to phylogeny and functional traits. We first quantified the species turnover in both forests. Both forests showed a substantial temporal decay in the species composition. The compositional turnover was greater in Luquillo, but the BCI forest also demonstrated a strong turnover through time (Appendix C).

The phylogenetic results show that on average the phylogenetic turnover in both forests has been random when accounting for the underlying levels of species turnover (Fig. 1). In other words, across the forest there was no clear directional trend in the phylogenetic turnover. In contrast to the phylogenetic results, the functional turnover of both forests has been highly nonrandom, but in opposing directions. In particular, the functional turnover in the BCI-FDP has been less than that expected given the underlying levels of species turnover while the functional turnover in the LUQ-FDP has been higher than expected given the underlying levels of species turnover (Fig. 1). In other words, the functional composition has been rather static in the BCI-FDP and directionally changing in the LUQ-FDP. This was confirmed by examining the forest-wide trait distributions in each of the two plots on a trait-by-trait basis (Fig. 2). This analysis shows little-to-no change in the forest-wide trait distributions in the BCI-FDP and significant shifts in the trait distributions in the LUQFDP through time. Thus for BCI-FDP, while there has been a large stem turnover through time, the new cohorts are more functionally similar to the stems that die than expected by chance. Conversely, the new cohorts in the LUQ-FDP have tended to be more functionally different from those stems that die than expected by chance.

\section{Habitat-specific phylogenetic and functional turnover}

A final goal of this paper was to examine whether rates of phylogenetic and functional turnover differ between habitat types in the two forest plots. Interestingly, while the phylogenetic turnover of the BCI-FDP is on average random phylogenetically, there was variation in the rate of turnover between habitats (Fig. 3). In particular, the "swamp" habitat of the BCI-FDP had a much higher rate of phylogenetic turnover while the "young" habitat had a much lower rate of turnover when compared to the other habitats suggesting that the phylogenetic turnover of the BCI-FDP is not completely random. The phylogenetic turnover of the LUQ-FDP, on the other hand, had only little differentiation between habitats.

The functional turnover in both forest plots was significantly different between multiple habitat type pairs (Figs. 1 and 3). Of particular interest is the finding that the young habitat in the BCI-FDP had an elevated rate of functional turnover similar to the values found in the LUQ-FDP. The analyses of the LUQ-FDP show that early on the more disturbed areas of the forest had very little functional turnover, but these areas rapidly turned over functionally from 2000 to 2005 (Figs. 1 and 3).

\section{DisCUSSION}

Discerning the mechanisms underlying the temporal dynamics of late- and early-successional plant communities is a major challenge in ecology. This is particularly true given the increasing number of secondary forests worldwide and future alterations to the climate. Hyperdiverse tropical tree communities present a vexing system for such research given their high biodiversity, small population sizes, and relatively slow temporal dynamics when compared to systems such as speciespoor temperate herbaceous communities. Further, mechanistic insights into community dynamics may not be possible by only quantifying the turnover of species names and abundances through time in absence of information pertaining to their function and general similarity to one another. Ultimately, studies of the long-term dynamics of tropical tree communities that incorporate information pertaining to species function and similarity hold perhaps the best chance of uncovering the mechanisms underlying their dynamics. Here we have presented such a study that has aimed to test whether the temporal turnover of two tropical forests has been deterministic by quantifying the degree to which their phylogenetic and functional turnover has been nonrandom.

The results of our analyses indicate that tropical tree community dynamics are governed primarily by deterministic factors in both of the forests studied. Specifically, functional composition of the relatively undisturbed BCIFDP subplots has, on average, turned over less than 


\section{Barro Colorado Island}

Phylogenetic turnover
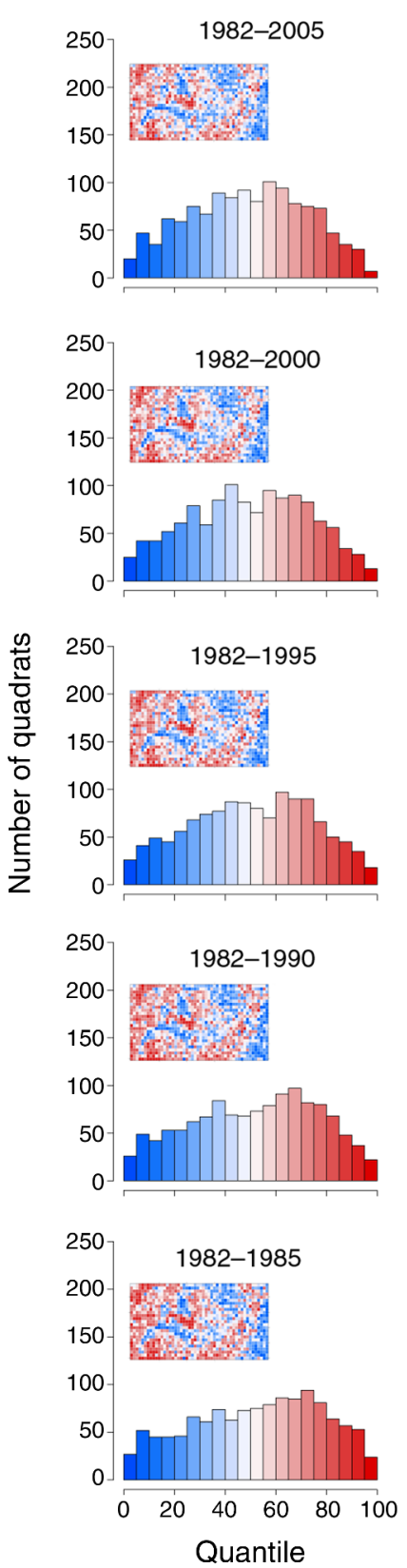

Functional turnover
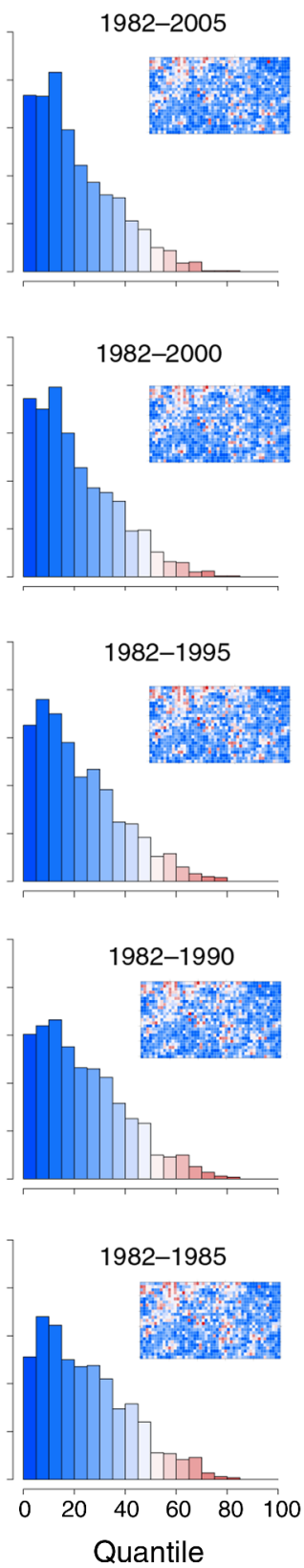

Luquilllo

Phylogenetic turnover

Functional turnover
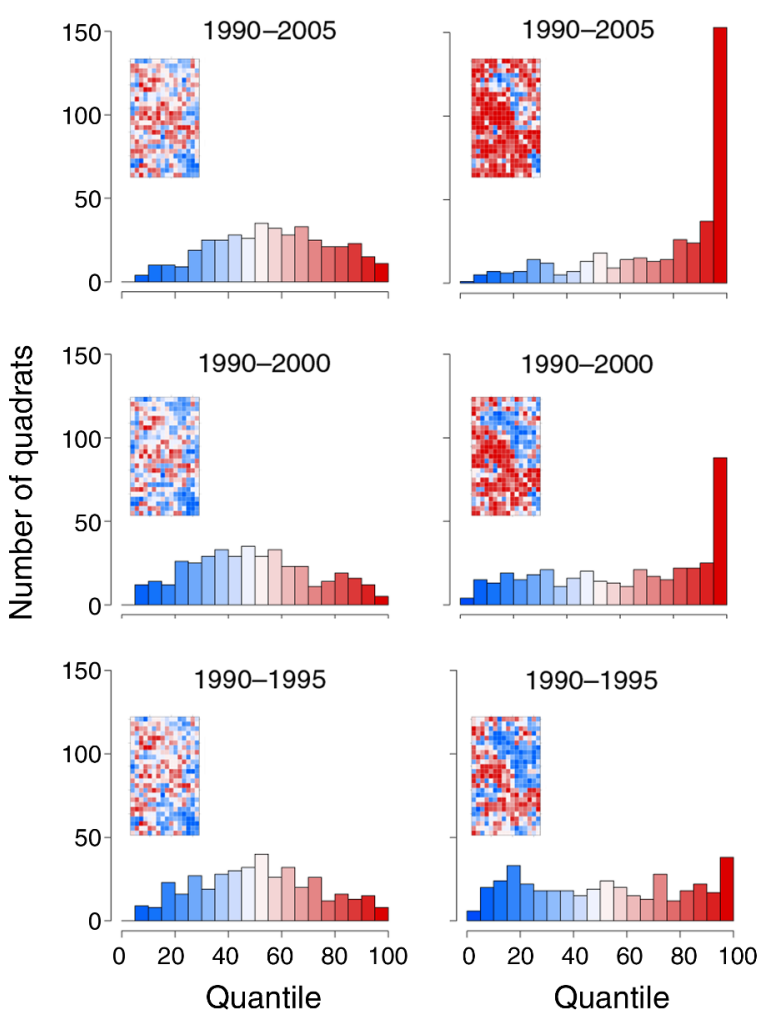

FIG. 1. Null model results for temporal turnover in the phylogenetic and functional composition of both forests (Barro Colorado Island Forest Dynamics Plot, Panama [BCI-FDP] and Luquillo Forest Dynamics Plot, Puerto Rico [LUQ-FDP]). Histograms represent quantile scores for the 1250 BCI-FDP subplots and the 400 LUQ-FDP subplots. Low quantile scores (blue) indicate lower than expected turnover in the phylogenetic or functional composition of the subplot, given the observed level of species turnover. High quantile scores (red) indicate higher than expected turnover in the phylogenetic or functional composition of the subplot, given the species turnover. Insets are maps of the forest dynamics plots where subplot colors follow the histogram color scheme. Maps are oriented on a north-south axis. Quantile scores provide a continuous measure of the magnitude of departure between observed and expected turnover; values $<2.5$ or $>97.5$ are significant at the 0.05 level. 

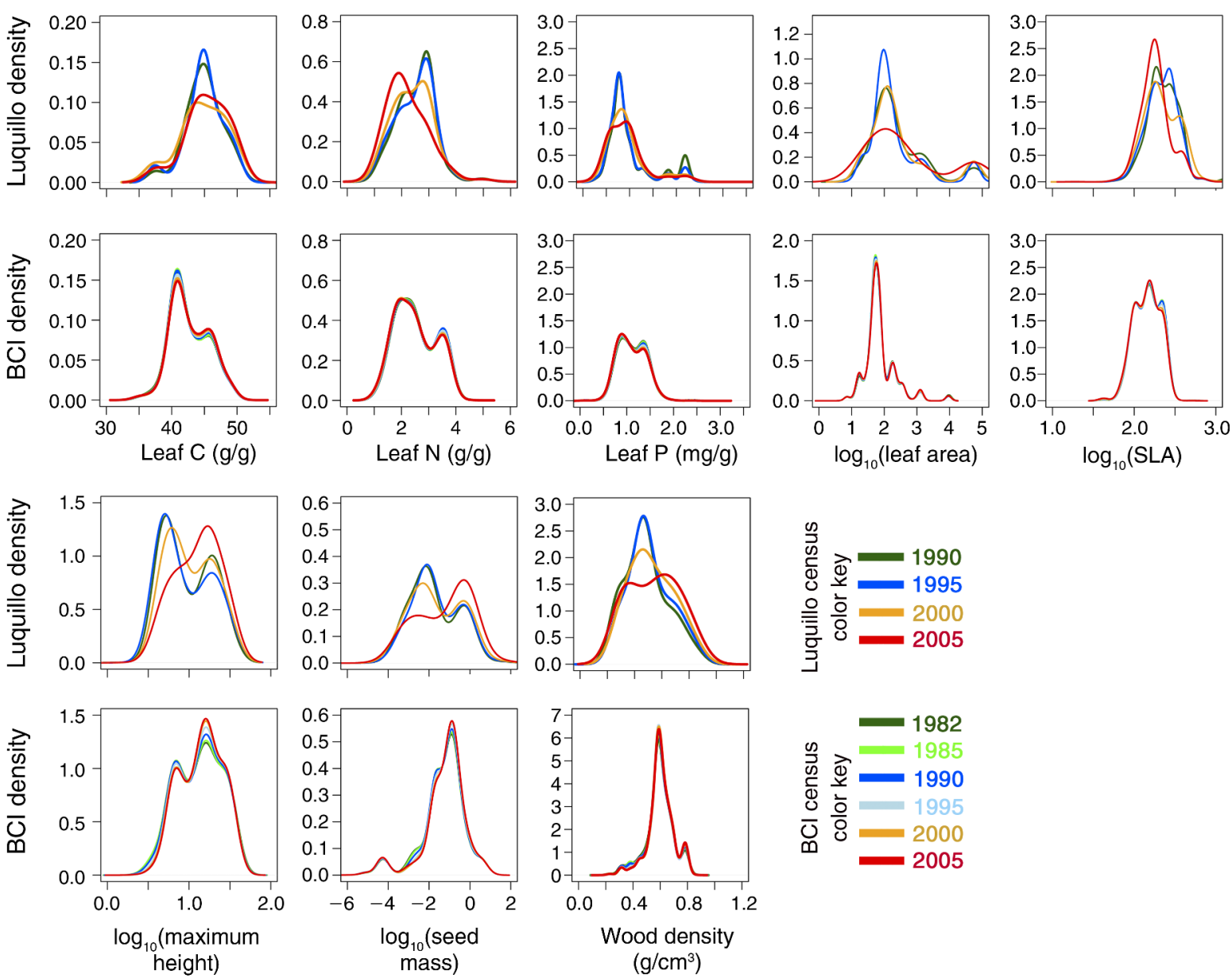

FIG. 2. Kernel density estimation for each functional trait in the two forest plots through time where the density is calculated by assigning a species mean trait value to all individuals in the forest plot. Different censuses have different colored lines. Note that lines for the BCI-FDP may not be distinguishable due to their high degree of overlap through time. Leaf area was originally measured in $\mathrm{cm}^{2}$; specific leaf area (SLA) was measured in $\mathrm{cm}^{2} / \mathrm{g}$; maximum height was measured in $\mathrm{m}$; seed mass was measured in $\mathrm{g}$.

expected given the amount of species turnover (Fig. 1). In other words, the functional composition of BCI-FDP subplots tended to be constrained through time, and the degree of this constraint appears to be increasing through time, as evidenced by a shift in the distribution of quantile scores to lower values as the time interval grows (Fig. 1). The results of the species turnover analyses do demonstrate that the species composition in BCI has indeed turned over through time (Appendix C), but our null modeling analyses demonstrated that the functional turnover has been less than that expected given this level of species compositional turnover. Last, when analyzing functional turnover in different BCI habitats (Harms et al. 2001), the young section of the forest that was previously disturbed by humans $\sim 110$ years ago had the highest levels of functional turnover when compared to habitats in older growth (Figs. 1 and 3; Appendix D).

In contrast to the BCI-FDP results, but also as predicted by a deterministic model, the functional composition of LUQ-FDP subplots has, on average, turned over more than expected given the amount of species turnover (Fig. 1). Humans disproportionately disturbed specific areas of the LUQ-FDP up until the 1940s, and these areas are known to be more prone to hurricane disturbance (Thompson et al. 2002). Specifically, natural disturbances primarily influence the populations of secondary forest species. These species were more common in areas that had suffered past human disturbance. Therefore existing human disturbance matrix plays a large role in explaining the posthurricane temporal dynamics in this forest (Thompson et al. 2002). Functional turnover is generally lower than expected initially after hurricanes Hugo and Georges in areas that were heavily disturbed by humans, but from 2000 to 2005 the functional turnover in these areas rapidly increased (Figs. 1 and 3; Appendix D). One potential explanation of the "lag" in functional turnover in human disturbed areas is that the seed bank in these areas may be heavily dominated by species adapted to disturbance. In turn, it may take a relatively long time 

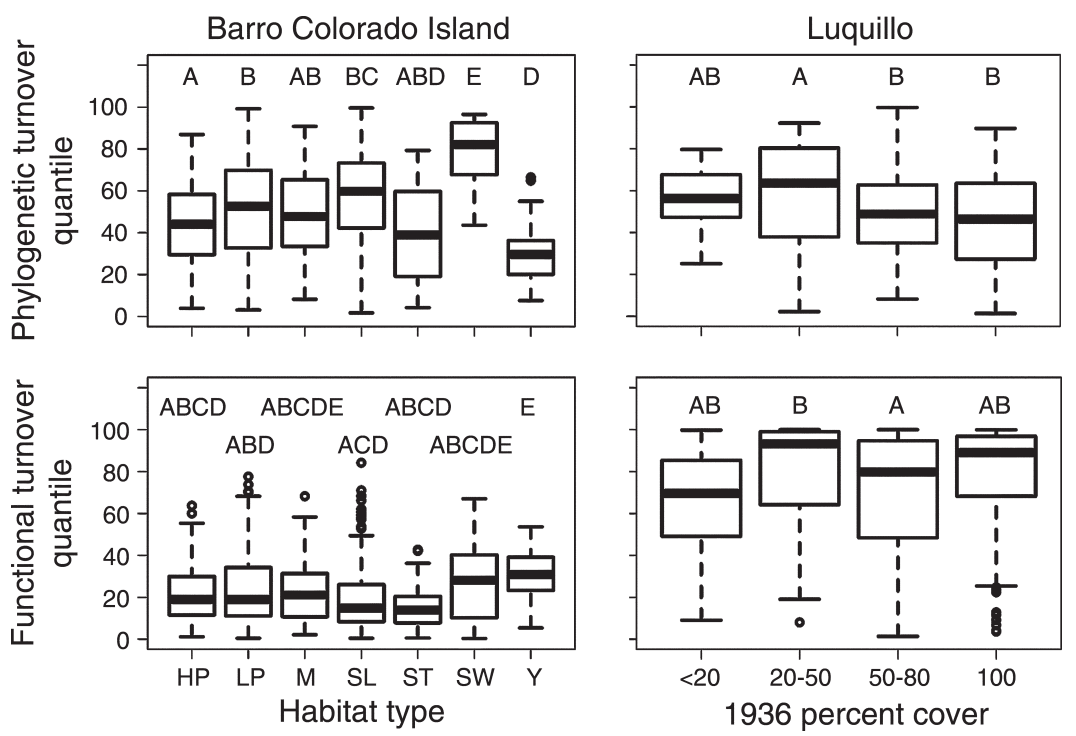

FIG. 3. Boxplots of the phylogenetic and functional turnover quantiles from the BCI-FDP for the 1982-2005 census interval in relation to the habitats described in Harms et al. (2001) and from the Luquillo FDP for the 1990-2005 census interval in relation to 1936 cover class (Thompson et al. 2002). Boxplots were made in R, and components are: top of the box, upper hinge; midline of box, median; bottom of box, lower hinge; bars, 1.5 times length of box (1.5 times the H-spread); dots, values that are greater or less than 1.5 times the H-spread of the distribution, plus the upper or lower hinge. Uppercase letters above the boxplots indicate results from Tukey tests; $P \leq 0.05$. All results from all census intervals are provided in the Appendices. Habitat types are: HP, high plateau; LP, low plateau; M, mixed; SL, slope; ST, stream; SW, swamp; Y, young.

for species not adapted to disturbance (and characterized by a different set of functional traits) to invade historically disturbed areas.

These nonrandom patterns of temporal turnover are further supported by evidence that forest-wide trait distributions in the BCI-FDP have shifted relatively little in comparison to the dramatic shifts in trait distributions in the LUQ-FDP, particularly between the 2000 and 2005 censuses (Fig. 2). The directional shift in these and other functional traits in the LUQ-FDP in particular support the expected trajectory of ecological strategies during succession. Specifically, short-lived shade-intolerant species, characterized by low wood densities, low seed masses, and high specific leaf areas, are expected to dominate initially after a disturbance (Grubb 1977, Bazzaz and Pickett 1980). Later, longlived shade-tolerant species, characterized by high wood densities, high seed masses, and low specific leaf areas are expected to dominate, consistent with the patterns observed here (Fig. 2).

The phylogenetic turnover has been, on average, random in both of the forests studied suggesting that unmeasured phylogenetically conserved traits may not play a large role in governing the dynamics of these two forests. Thus, if tropical tree defense traits do have phylogenetic signal, as recent research has suggested (Gilbert and Webb 2007), then these traits may be of minimal importance in driving the temporal dynamics of the two forests studied. That said, the phylogenetic turnover in the BCI-FDP was not spatially random with different habitats having significantly different levels of phylogenetic turnover (Figs. 1 and 3; Appendix D). It is difficult to determine what may be driving these spatial patterns, but it is clear that the rates of phylogenetic turnover between habitats have varied significantly. One may speculate that a trait or series of traits that have phylogenetic signal are important in determining the temporal dynamics in particular habitats and not in others, but the present work has no evidence to test this prediction. Ultimately, further work will be needed to identify the causal factors underlying this spatial pattern and more importantly additional investigations into the phylogenetic signal in defense traits and natural enemies is certainly needed. Unlike the BCI-FDP, the LUQ-FDP had very little spatial structure in its rates of phylogenetic turnover (Figs. 1 and 3; Appendix D) suggesting that the measured traits, which have very little phylogenetic signal (Table 1), are potentially the principal drivers of the dynamics of the tree community.

\section{LiMITATIONS}

The present work has uncovered clear trait-based evidence of deterministic temporal turnover of the two tropical forests studied. That said, there are a few key limitations of the work to highlight. First, the present work was only able to analyze two forest plots on very different ends of the successional spectrum. Additional study sites on both ends of this spectrum and in between would help determine the generality of the results presented. Second, it is naïve to believe that the few simple traits measured in this study are the only aspects of plant function determining community structure and 
dynamics. Given the large number of species in tropical forests it has proven difficult to perform complex physiological measurements on multiple individuals in each species in entire communities and to strongly consider intraspecific variability. We suspect that this will continue to be an issue, but in locations such as Luquillo and BCI with large numbers of researchers these obstacles may be overcome. Last, the present study only considers individuals $>1 \mathrm{~cm}$ in diameter and does not address the dynamics of seedling communities where different traits or processes may become more important. Similar research to that presented here performed on tropical seedling plots would therefore be highly valuable.

\section{Conclusions}

Here by directly quantifying the functional and phylogenetic composition of communities through time in a comparative analysis we have provided evidence of nonrandom temporal turnover in the species composition of two tropical forest dynamics plots. These results reject a model based purely on stochastic factors such as ecological drift, reject a model based purely on more closely related species sharing more pathogens, and support a model in which community dynamics are primarily governed by the fit of individuals to their abiotic environment. The dominance of ecological filtering suggests that it may be possible to predict the future composition of tropical forests with diverse disturbance histories on the basis of plant function.

\section{ACKNOWLEDGMENTS}

N. G. Swenson and J. C. Stegen contributed equally to this paper. We thank R. Condit for discussions regarding the dynamics of the BCI forest dynamics plot. The collection of plant material for DNA sequencing and trait quantification was funded by the F. H. Levinson Fund for BCI and a Center for Tropical Forest Science research grant awarded to N. G. Swenson for Luquillo. DNA barcode sequencing and analyses were supported by funds from the Smithsonian Institution Global Earth Observatories project. The Luquillo forest plot has been supported by National Science Foundation grants (BSR-9015961 and DEB-0516066) and from the Mellon Foundation. National Science Foundation funds to the Luquillo Long-Term Ecology Research Site (BSR-88111902, DEB-9411973, DEB-008538, DEB-0218039, and DEB0620910) also supported this work. The BCI forest dynamics research project was made possible by National Science Foundation grants to Stephen P. Hubbell: DEB-0640386, DEB-0425651，DEB-0346488，DEB-0129874，DEB-00753102, DEB-9909347, DEB-9615226, DEB-9615226, DEB-9405933, DEB-9221033，DEB-9100058，DEB-8906869，DEB-8605042, DEB-8206992, DEB-7922197, support from the Center for Tropical Forest Science, the Smithsonian Tropical Research Institute, the John D. and Catherine T. MacArthur Foundation, the Mellon Foundation, the Celera Foundation, numerous private individuals, and through the hard work of over 100 people from 10 countries over the past two decades. Both forest plots are part of the Center for Tropical Forest Science, a global network of large-scale demographic tree plots. J. C. Stegen was supported by an NSF Postdoctoral Fellowship in Bioinformatics (DBI-0906005).

\section{Literature Cited}

Bazzaz, F. A., and S. T. A. Pickett. 1980. Physiological ecology of tropical succession: a comparative review. Annual Review of Ecology and Systematics 11:287-310.

Binida-Edmonds, O. R. P. 2005. transAlign: using amino acids to facilitate the multiple alignment of protein-coding DNA sequences. BMC Bioinformatics 6:156.

Blomberg, S. P., T. Garland, Jr., and A. R. Ives. 2003. Testing for phylogenetic signal in comparative data: behavioral traits are more labile. Evolution 57:717-745.

Chase, J. M., and M. A. Leibold. 2003. Ecological niches: linking classical and contemporary approaches. University of Chicago Press, Chicago, Illinois, USA.

Chave, J., D. Coomes, S. Jansen, S. Lewis, N. G. Swenson, and A. E. Zanne. 2009. Towards a worldwide wood economics spectrum. Ecology Letters 12:351-366.

Condit, R. 1998. Tropical forest census plots. Springer-Verlag, Berlin, Germany.

Connell, J. H. 1971. On the role of natural enemies in preventing competitive exclusion in some marine animals and in rainforest trees. Pages 298-312 in P. J. Den Boer and G. Gradwell, editors. Dynamics of populations. PUDOC, Wageningen, The Netherlands.

Cornelissen, J. H. C., S. Lavorel, E. Garnier, S. Díaz, N. Buchmann, D. E Gurvich, P. B. Reich, H. ter Steege, H. D. Morgan, M. G. A. van der Heijden, J. G. Pausas, and H. Poorter. 2003. A handbook of protocols for standardised and easy measurement of plant functional traits worldwide. Australian Journal of Botany 51:335-380.

Edgar, R. 2004. MUSCLE: multiple sequence alignment with high accuracy and high throughput. Nucleic Acids Research 32:1792-1797.

Gilbert, G. S., and C. O. Webb. 2007. Phylogenetic signal in plant pathogen-host range. Proceedings of the National Academy of Sciences USA 104:4979-4983.

Gower, J. C. 1971. A general coefficient of similarity and some of its properties. Biometrics 27:857-874.

Grubb, P. J. 1977. The maintenance of species-richness in plant communities: the importance of the regeneration niche. Biological Reviews 52:107-145.

Hardy, O. J. 2008. Testing the spatial phylogenetic structure of local communities: statistical performances of different null models and test statistics on a locally neutral community. Journal of Ecology 96:914-926.

Harms, K. E., R. Condit, S. P. Hubbell, and R. B. Foster. 2001 Habitat associations of trees and shrubs in a 50-ha neotropical forest plot. Journal of Ecology 89:947-959.

Hubbell, S. P. 2001. The unified neutral theory of biodiversity and biogeography. Princeton University Press, Princeton, New Jersey, USA.

Hubbell, S. P., R. Condit, and R. B. Foster. 2005. Barro Colorado Forest Census Plot Data. http://ctfs.arnarb. harvard.edu/webatlas/datasets/bci/

Hubbell, S. P., R. B. Foster, S. T. O'Brien, K. E. Harms, R. Condit, B. Wechsler, S. J. Wright, and S. Loo de Lao. 1999. Light-gap disturbances, recruitment limitation, and tree diversity in a neotropical forest. Science 283:554-557.

Janzen, D. H. 1970. Herbivores and the number of tree species in tropical forests. American Naturalist 104:501-528.

Kress, W. J., D. L. Erickson, F. A. Jones, N. G. Swenson, R. Perez, O. Sanjur, and E. Bermingham. 2009. Plant DNA barcodes and a community phylogeny of a tropical forest dynamics plot in Panama. Proceedings of the National Academy of Sciences USA 106:18621-18626.

Kress, W. J., D. L. Erickson, N. G. Swenson, J. Thompson, M. Uriarte, and J. K. Zimmerman. 2010. Advances in the use of DNA barcodes to build a community phylogeny for tropical trees in a Puerto Rican forest dynamics plot. PLoS One 5:e15409. 
Levine, J. M., and J. HilleRisLambers. 2009. The importance of niches for the maintenance of species diversity. Nature 461:254-257.

Moles, A. T., D. I. Warton, L. Warman, N. G. Swenson, S. W. Laffan, A. E. Zanne, A. Pitman, F. A. Mennings, and M. R. Leishman. 2009. Global patterns in plant height. Journal of Ecology 97:923-932.

Piperno, D. R. 1989. Fitolitos, arqueologia y cambios prehistoricos de la vegetacion en un lote de cincuenta hectares de la isla de Barro Colorado. Pages 153-156 in E. G. Leigh, Jr., A. S. Rand, and D. M. Windsor, editors. Ecologia de un bosque tropical. Smithsonian Tropical Research Institute, Balboa, Panama.

R Development Core Team. 2010. R: a language and environment for statistical computing. R Foundation for Statistical Computing, Vienna, Austria. http://www. R-project.org

Swenson, N. G. 2011a. The role of evolutionary processes in producing biodiversity patterns, and the interrelationships between taxonomic, functional and phylogenetic biodiversity. American Journal of Botany 98:472-480.

Swenson, N. G. 2011 b. Phylogenetic beta diversity metrics, trait evolution and inferring the functional beta diversity of communities. PLoS One 6:e21264.

Swenson, N. G., P. Anglada-Cordero, and J. A. Barone. 2011. Deterministic tropical tree community turnover: evidence from patterns of functional beta diversity along an elevational gradient. Proceedings of the Royal Society B 278:877884.

Swenson, N. G., and B. J. Enquist. 2007. Ecological and evolutionary determinants of a key plant functional trait: wood density and its community-wide variation across latitude and elevation. American Journal of Botany 91:451-459.

Swenson, N. G., and B. J. Enquist. 2008. The relationship between stem and branch wood specific gravity and the ability of each measure to predict leaf area. American Journal of Botany 95:516-519.
Swenson, N. G., and B. J. Enquist. 2009. Opposing assembly mechanisms in a Neotropical dry forest: implications for phylogenetic and functional community ecology. Ecology 90:2161-2170.

Swenson, N. G., B. J. Enquist, J. Pither, J. Thompson, and J. K. Zimmerman. 2006. The problem and promise of scale dependency in community phylogenetics. Ecology 87:2418 2424.

Swenson, N. G., B. J. Enquist, J. Thompson, and J. K. Zimmerman. 2007. The influence of spatial and size scales on phylogenetic relatedness in tropical forest communities. Ecology 88:1770-1780.

Swofford, D. L. 2003. PAUP*. Phylogenetic analysis using parsimony (*and other methods). Version 4. Sinauer Associates, Sunderland, Massachusetts, USA.

Thompson, J., N. Brokaw, J. K. Zimmerman, R. B. Waide, E. M. Everham III, D. J. Lodge, C. M. Taylor, D. GarciaMontiel, and M. Fluet. 2002. Land use history, environment, and tree composition in a tropical forest. Ecological Applications 12:1344-1363.

Webb, C. O. 2000. Exploring the phylogenetic structure of ecological communities: an example for rain forest trees. American Naturalist 156:145-155.

Westoby, M., D. S. Falster, A. T. Moles, P. A. Vesk, and I. J. Wright. 2002. Plant ecological strategies: some leading dimensions of variation between species. Annual Review of Ecology and Systematics 33:125-159.

Wright, S. J. 2002. Plant diversity in tropical forests: a review of mechanisms of species coexistence. Oecologia 130:1-14.

Wright, S. J., et al. 2010. Functional traits and the growthmortality trade-off in tropical trees. Ecology 91:3664-3674.

Zimmerman, J. K., L. S. Comita, J. Thompson, M. Uriarte, and N. Brokaw. 2010. Patch dynamics and community metastability of a subtropical forest: compound effects of natural disturbance and human land use. Landscape Ecology 25:1099-1111.

\section{Supplemental Material}

Appendix A

The results of the PCA analyses of functional traits (Ecological Archives E093-046-A1).

\section{Appendix B}

Maps of habitat types in each forest plot (Ecological Archives E093-046-A2).

\section{Appendix C}

Temporal turnover of species composition results (Ecological Archives E093-046-A3).

Appendix D

The results of the habitat analyses (Ecological Archives E093-046-A4). 